\title{
823 SPATIAL ANALYSIS OF TUMOR-INFILTRATING LYMPHOCYTES CORRELATES WITH THE RESPONSE OF METASTATIC COLORECTAL CANCER PATIENTS TREATED WITH VACTOSERTIB IN COMBINATION WITH PEMBROLIZUMAB
}

\begin{abstract}
${ }^{1}$ Tae Won Kim*, ${ }^{2}$ Keun-Wook Lee, ${ }^{3}$ Joong Bae Ahn, ${ }^{4}$ Young Suk Park, ${ }^{5}$ Gahee Park, ${ }^{5}$ Kyunghyun Paeng, ${ }^{5}$ Chan-Young Ock, ${ }^{6}$ Hyejoo Park, ${ }^{6}$ Jiyeon Ryu, ${ }^{6}$ Bitna Oh, ${ }^{6}$ BoKyoung Kim, ${ }^{6}$ Sunjin Hwang, ${ }^{6}$ Ki Baik Hahm, ${ }^{6}$ Seong-Jin Kim. ${ }^{1}$ Asan Medical Center, University of Ulsan College of Medicine, Seoul, Korea, Republic of; ${ }^{2}$ Seoul National University Bundang Hospital, Seoul National University College of Medicine, Seoul, Korea, Republic of; ${ }^{3}$ Yonsei Cancer Center, Yonsei University College of Medicine, Seoul, Korea, Republic of; ${ }^{4}$ Samsung Medical Center, Sungkyunkwan University School of Medicine, Seoul, Korea, Republic of; ${ }^{5}$ Lunit Inc., Seoul, Korea, Republic of; ${ }^{6}$ MedPacto, Inc., Seoul, Korea, Republic of
\end{abstract}

Background Previously, we presented a promising anti-tumor efficacy (ORR: 16\%, mOS: 15.8 months, RECIST) of the combination of vactosertib, a potent and selective TGF- $\beta$ receptor I, and pembrolizumab (vac+pem) in patients with microsatellite stable metastatic colorectal cancer (MSS mCRC, MP-VAC-204 study). Recent reports showed immune-excluded TIL located in stroma would be closely related to TGF- $\beta$ signature, which may harbor the primary resistance of pembrolizumab. In this study, we performed an exploratory biomarker analysis of TIL resided in either intra-tumoral or stromal area in pathology slides, and we hypothesized that spatial features of TIL would correlate with the response of vac+pem.

Methods Pathology slides stained with H\&E were obtained from 31 patients at baseline and 14 patients at cycle 2 in MSS mCRC patients in MP-VAC-204 study. For spatial TIL analysis, we applied an artificial intelligence -powered H\&E analyzer, named Lunit SCOPE IO, which automatically detects TIL, tumor and stroma. It calculates the proportion of immune phenotype consists of inflamed, as high TIL density inside tumor area, or immune-excluded, as high TIL density in stroma in whole-slide images. Additionally, PD-L1 and CD8 were stained using multiplex immunohistochemistry to validate immune phenotype assessed by Lunit SCOPE IO.

Results At baseline, the proportion of immune-excluded area (immune-excluded score, IES) was positively correlated with the density of CD8-positive cells in stroma area measured by mIHC (coefficient $=0.349$ ), but it was not related to the density of PD-L1-positive cells (coefficient $=-0.226$ ). Area under receiver operating characteristics to predict the responder as partial response by RECIST v1.1 by IES and PD-L1 were 0.741 and 0.528 . The overall response rate of vac + pem in the patients with high IES $>42.3 \%$ was $25 \%$ (4 out of 16), while no response was observed in those with low IES (0 out of 15). Overall survival (OS) of vac+pem was significantly prolonged in those with high IES $>42.3 \%$ compared to low IES (median OS: not reached versus 6.8 months, $\mathrm{P}=$ 0.0097), but it was not different according to PD-L1 level. After treatment of vac+pem, while IES was decreased regardless of treatment response, the proportion of inflamed area was increased in the responders $(\mathrm{N}=3)$ but decreased in the non-responders $(\mathrm{N}=11)$.

Conclusions Immune-excluded score which reflects TGF- $\beta$ driven TIL exclusion into stroma is correlated with anti-tumor response of $v a c+$ pem in MSS mCRC. Further investigation on spatial TIL analysis as a potential biomarker should be warranted. (Clinical trial information: NCT03724851)

http://dx.doi.org/10.1136/jitc-2021-SITC2021.823 